\title{
Rapid fluid administration: an evaluation of two techniques
}

This article was published in the following Dove Press journal:

Medical Devices: Evidence and Research

\author{
Holly C Gillis' \\ Hina Walia ${ }^{2}$ \\ Dmitry Tumin ${ }^{2,3}$ \\ Tarun Bhalla ${ }^{2,3}$ \\ Joseph D Tobias ${ }^{1-4}$
}

'Department of Pediatrics, Division of Critical Care Medicine, Nationwide Children's Hospital, Columbus, $\mathrm{OH}$, USA; ${ }^{2}$ Department of Anesthesiology \& Pain Medicine, Nationwide Children's Hospital, Columbus, $\mathrm{OH}$, USA; ${ }^{3}$ Department of Pediatrics, The Ohio State University College of Medicine, Columbus, OH, USA; ${ }^{4}$ Department of Anesthesiology \& Pain Medicine, The Ohio State University College of Medicine, Columbus, $\mathrm{OH}$, USA

Correspondence: Holly Catherine Gillis, MD

Department of Pediatrics, Division of Critical Care Medicine, Nationwide Children's Hospital, 700 Children's Drive, Columbus, Ohio 43205, USA

$\mathrm{Tel}+\mathrm{I}(6 \mathrm{I} 4) 722-4200$

Fax +I (6I4) 722-4203

Email holly.gillis@nationwidechildrens.org
Objective: Rapid administration of fluid remains a cornerstone in treatment of shock and when caring for trauma patients. A range of devices and technologies are available to hasten fluid administration time. While new devices may optimize fluid delivery times, impact on subjective experience compared to traditional methods is poorly documented. Our study evaluated administration time and provider experience using two unique methods for fluid administration. Materials and methods: Prospective comparison of objective and subjective outcomes using a novel infusion device (LifeFlow ${ }^{\circledR}$ Rapid Infuser) and the traditional push-pull syringe method in a simulated model of rapid fluid infusion. Ten paired trials were conducted for each of three intravenous catheter gauges. Providers administered $500 \mathrm{~mL}$ of isotonic crystalloid through an intravenous catheter with both LifeFlow and a push-pull device. Administration time was compared between devices using paired $t$-tests. Participants' subjective physical demand, effort, pain, and fatigue using each device were recorded using 21-point visual analog scales and compared between devices using sign-rank tests.

Results: Fluid administration time was significantly decreased with LifeFlow compared to the push-pull device with the 18 -gauge catheter $(2.5 \pm 0.8$ vs $3.8 \pm 1.0$ minutes; $95 \%$ CI of difference: $0.9,1.8$ minutes; $P<0.001)$. Findings were similar for other catheter sizes. No improvements in subjective experience were noted with the LifeFlow device. Increased physical demand with the LifeFlow device was noted with 18 and 22 gauge catheters, and increased fatigue with the LifeFlow device was noted for all catheter sizes.

Conclusion: The LifeFlow device was faster than the push-pull syringe method in our simulated scenario. However, provider subjective experience was not improved with the LifeFlow device

Keywords: Resuscitation, Shock, Trauma, Hemodynamics, Crystalloid Solutions

\section{Introduction}

Regardless of the clinical scenario, the rapid administration of fluid may be indicated to restore intravascular volume, augment cardiac output, and reverse shock. ${ }^{1-3}$ If not rapidly and effectively treated, progressive decompensation will occur, resulting in death. Early and aggressive fluid therapy has been shown to decrease morbidity and mortality. ${ }^{4-6}$ Resuscitation guidelines from various organizations recommend the rapid intravascular administration of isotonic crystalloid as a key component of the initial resuscitation process. ${ }^{7-10}$ Various techniques and devices have been recommended for the rapid administration of fluid. ${ }^{11-14}$ Still, more rapid options are needed to facilitate faster administration of fluid to augment hemodynamics in acute clinical scenarios and expedite administration of further fluid or initiation of vasopressors. A commonly used technique in pediatric resuscitation scenarios is a simple syringe, stopcock, and 
tubing setup known as the "push-pull method" whereby fluids are intermittently drawn into a syringe from the infusion bag and then manually administered to the patient by turning the stopcock. ${ }^{15}$

The LifeFlow ${ }^{\circledR}$ Rapid Infuser (410 Medical, Durham, NC, USA) is a single-use, hand-operated device designed to allow a health care provider to quickly and efficiently deliver recommended fluid volumes. The device has a $10 \mathrm{~mL}$ syringe with graduated markings that are visible through the transparent canopy of the device (Figure 1). It automatically recoils and refills with fluid when the trigger is released. LifeFlow can be used with cannulas of various sizes down to 24 gauge. The current study prospectively compared the commonly used "push-pull" systems with the novel LifeFlow device. Our primary hypothesis was that the use of the LifeFlow device would reduce the time needed to administer $500 \mathrm{~mL}$ of fluid, compared to the push-pull method. Our secondary aim was to determine whether use of the LifeFlow device would reduce subjective effort or fatigue associated with rapid fluid administration.

\section{Materials and methods}

The study was a prospective, nonblinded observational simulated trial that did not involve patient care. Trials were performed by anesthesia providers including anesthesiology faculty, fellows and residents, as well as certified registered nurse anesthetists (CRNA) and student registered nurse anesthetists (SRNAs) from a single institution. Fluid bags were connected to tubing and subsequently either to a push-pull setup or the LifeFlow device. From either setup, subsequent tubing was connected to intravenous (IV) catheters in various sizes as described below. The trials occurred over 6 hours on 3 days with 1 day assigned for each size of catheter. Based on the design of similar studies investigating time to deliver a fixed fluid quantity with various experimental setups, ten trials were performed for each of three different IV catheter sizes (18, 20, and 22 gauge). ${ }^{15,16}$ The sample size of ten trials was deemed to have at least $87 \%$ power to detect a previously reported improvement in fluid administration time from $151 \pm 22$ seconds with a push-pull setup to $130 \pm 11$ seconds with the use of a hand pump at a $95 \%$ confidence level, assuming moderate $(r=0.5)$ correlation in performance between trials by the same provider. For each trial, providers were instructed to empty as rapidly as possible a $500 \mathrm{~mL}$ bag of normal saline solution that was hanging $1.8 \mathrm{~m}$ above the ground on an IV pole. Providers were instructed to perform this task as they would in the event of an intraoperative emergency or in a trauma patient. Each provider performed the trial with both the LifeFlow or push-pull systems through

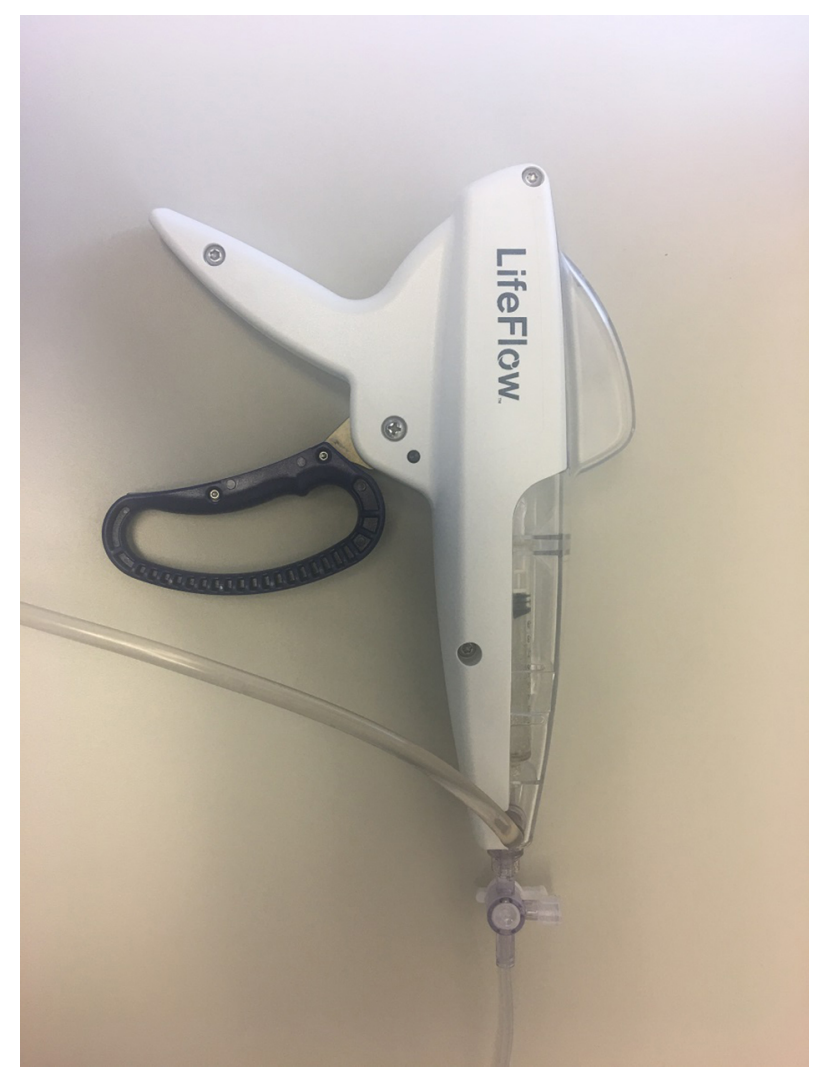

Figure I Photograph of the LifeFlow ${ }^{\circledR}$ Rapid Infuser used in the current study. Note: Although there is proprietary tubing to connect the device to the intravenous cannula, we chose to use a 33 inch extension tubing with a three-way $\mathrm{Hi}$-Flo stopcock to connect the device to the intravenous cannula to keep the tubing standardized across the two delivery systems.

an IV cannula of the same size. The sequence of devices was randomly alternated for each catheter size, and trials with different catheter sizes were conducted on separate days.

For both systems, fluid was administered through 18, 20, and 22-gauge IV catheters (Becton Dickinson, Franklin Lakes, NJ, USA). Although the LifeFlow system is meant for single patient use, for the purpose of this study, the devices were used several times. The LifeFlow system with its proprietary $10 \mathrm{~mL}$ syringe was connected to the $500 \mathrm{~mL}$ fluid bag using the manufacturer-provided tubing setup. As the tubing exited the device, it was attached to a piece of extension tubing with a three-way Hi-Flo stopcock and a 33 inch extension (Smiths Medical, Dublin, OH, USA). The 33 inch extension piece with stopcock was also used in the push-pull system. The extension tubing was connected to the IV cannula. For the push-pull system, Clearlink latex free, Y-type blood tubing was used (Hospira, Lake Forest, IL, USA) and a three-way Hi-Flo stopcock with a 33 inch extension and a standard 10 $\mathrm{mL}$ luer-lock syringe (Becton Dickinson) was connected to the 
tubing. For both setups, the IV cannula was taped over a sink and the system primed with fluid prior to the start of the timer.

Fluid administration time was recorded by a research assistant from the time the provider received a verbal direction to start, until the bag was emptied. After completing each trial, providers described the level of physical demand of administering the fluid, the effort required to use the setup, and the fatigue and the pain associated with operating the setup. Twenty-one point visual analog scale (VAS, from $1=$ lowest to 21=highest) was used for consistency with the Task Load Index tool for subjective evaluation of workload. ${ }^{17}$ Data were grouped by catheter size for analysis. Comparisons by device type were made using each provider as their own control. Fluid administration times were compared according to device type using paired $t$-tests and VAS responses were compared according to device type using nonparametric sign-rank tests. No adjustment for multiple comparisons was performed because of the exploratory nature of the study. Data analysis was completed in Stata/IC 14.2 (StataCorp LP, College Station, TX, USA), and two-tailed $P<0.05$ was considered statistically significant.

\section{Results}

A total of 62 trials were initiated, of which 31 were completed with a push-pull device and 30 with a LifeFlow device. One provider failed to complete the trial with the LifeFlow device as the handle broke during the trial. This provider had already successfully completed a trial with the push-pull device. Results from the latter trial were excluded from the analysis. In the remaining 60 trials, each trial with the push-pull device was paired with a trial using the LifeFlow device that was performed by the same provider through the same sized IV cannula. On paired $t$-test analysis, fluid administration time was significantly decreased with the LifeFlow compared to the push-pull device with the 18 gauge $(2.5 \pm 0.8$ vs $3.8 \pm 1.0$ minutes; 95\% CI of difference: $0.9,1.8$ minutes; $P<0.001$ ), 20 gauge $(2.8 \pm 0.5$ vs $3.8 \pm 0.6$ minutes; $95 \% \mathrm{CI}$ of difference: $0.6,1.4$ minutes; $P<0.001$ ), and 22 gauge ( $3.3 \pm 0.8$ vs $5.3 \pm 1.7$ minutes; 95\% CI of difference: 1.1, 2.8 minutes; $P<0.001)$ catheters (Table 1). There were no improvements in subjective reports of physical demand, effort, pain, or fatigue when comparing the LifeFlow device to the push-pull setup (Table 1). Rather, subjective physical demand on a 21-point scale (1= lowest, $21=$ highest) was somewhat greater with the LifeFlow device than with the push-pull system in the trials involving 18-gauge catheters (LifeFlow median $=13$, push-pull median $=10, P=0.048$ ) and in the trials involving 22-gauge catheters (LifeFlow median $=15$, push-pull median $=12, P=0.006$ ).

\section{Discussion}

The current simulation study demonstrates that fluid administration is significantly faster with the novel LifeFlow Rapid

Table I Fluid administration times and subjective participant ratings of the infusion devices

\begin{tabular}{|c|c|c|c|}
\hline \multirow[t]{2}{*}{ Catheter size and study outcome } & \multirow{2}{*}{$\begin{array}{l}\text { Push-pull system } \\
\text { Mean (SD) or median (IQR) }\end{array}$} & \multirow{2}{*}{$\frac{\text { LifeFlow }^{\circledR}}{\text { Mean (SD) or median (IQR) }}$} & \multirow[t]{2}{*}{$P$-value } \\
\hline & & & \\
\hline \multicolumn{4}{|l|}{18 gauge $n=10$} \\
\hline Fluid administration time (minutes) ${ }^{\mathrm{b}}$ & $3.8(1.0)$ & $2.5(0.8)$ & $<0.001$ \\
\hline Physical demand ${ }^{c}$ & $10(8,12)$ & $13(10,15)$ & 0.048 \\
\hline Effort $^{c}$ & II $(8,15)$ & $15(10,18)$ & 0.100 \\
\hline Fatigue $^{c}$ & $8(1,10)$ & II $(5,15)$ & 0.035 \\
\hline Pain $^{c}$ & $5(1,10)$ & $9(3,17)$ & 0.265 \\
\hline \multicolumn{4}{|l|}{20 gauge $n=10$} \\
\hline Fluid administration time (minutes) $^{\mathrm{b}}$ & $3.8(0.6)$ & $2.8(0.5)$ & $<0.001$ \\
\hline Physical demand ${ }^{c}$ & $14(8,18)$ & $13(12,16)$ & 0.798 \\
\hline Effort $^{c}$ & $15(7,18)$ & $14(12,17)$ & 0.573 \\
\hline Fatigue $^{c}$ & $6(5,14)$ & $12(8,15)$ & 0.082 \\
\hline Pain $^{c}$ & $7(5,10)$ & $8(5,15)$ & 0.608 \\
\hline \multicolumn{4}{|l|}{22 gauge $n=10$} \\
\hline Fluid administration time (minutes) ${ }^{\mathrm{b}}$ & $5.3(1.7)$ & $3.3(0.8)$ & $<0.001$ \\
\hline Physical demand ${ }^{c}$ & $12(10,17)$ & $15(15,18)$ & 0.006 \\
\hline Effort $^{c}$ & $15(8,18)$ & $16(14,18)$ & 0.182 \\
\hline Fatigue $^{c}$ & $9(4,12)$ & $14(5,18)$ & 0.081 \\
\hline Pain ${ }^{c}$ & $6(4,12)$ & II $(5,12)$ & 0.152 \\
\hline
\end{tabular}

Notes: ${ }^{\mathrm{F}}$ For each catheter size, ten participants performed the trial with both devices for each catheter size. ${ }^{\mathrm{b}}$ Time to administer $500 \mathrm{~mL}$ of normal saline. ${ }^{\mathrm{C}} \mathrm{Reported}$ by participants on I-2I scale from lowest to highest.

Abbreviation: IQR, interquartile range. 
Infuser than with the commonly used push-pull system. In the nonemergent scenario, fluids are generally administered by gravity in the operating room setting or by an infusion device in the inpatient setting. Neither is effective when the rapid administration of fluid is required for resuscitation. An infusion pump set at the maximum rate of $999 \mathrm{~mL} /$ hour delivers only $16-17 \mathrm{~mL}$ of fluid per minute, which is far below the rate of approximately $132 \mathrm{~mL} /$ minute with the push-pull system or $200 \mathrm{~mL} /$ minute with the infusion device through 18-gauge IV catheters. To our knowledge, ours is the first study comparing this device to other techniques commonly used in the pediatric population to rapidly administer fluid, including the use of a syringe for a push-pull system. Extrapolation of our simulated data would suggest that a $20 \mathrm{~mL} / \mathrm{kg}$ bolus could be administered to a $20 \mathrm{~kg}$ patient in approximately 130-135 seconds through a 20-gauge cannula with the LifeFlow device. This implies a faster time to administer the fluid than the 5 minutes recommended by resuscitation guidelines, and a faster rate than previously reported when using the push-pull system or a pressure bag to augment flow. ${ }^{18}$ The rapid administration of fluid is paramount for management of hemorrhagic and distributive shock as well as, in smaller volumes, for the management of cardiogenic shock. The more rapidly this fluid is delivered, the more faster the clinician can move on to other opportunities for intervention including vasopressor initiation and other medication administration.

Regardless of the device or technique used, flow rates and administration times are also affected by the resistance of the infusion line and cannula as well as the viscosity of the fluid. ${ }^{15}$ As noted in our study, administration times increased with smaller gauge cannulas as resistance increases to the fourth power with a decrease in radius, and increases linearly with the length of the cannula or infusion tubing. ${ }^{19,20}$ Resistance beyond the cannula may also impact fluid administration rates. This resistance was not present in our simulated model as the cannula emptied into a sink. Additionally, given the impact of viscosity on flow based on Poiseuille's law, administration rates decrease significantly when administering blood vs crystalloid. It should be noted that the LifeFlow has not been rigorously evaluated for the infusion of blood, blood products, and albumin. It currently does not have approval by the United States Food and Drug Administration for such use.

Limitations to our study include the use of a simulated model. However, use of this model in a controlled setting allowed us to exclude the influence of other factors that impact fluid administration times. User training and experience with various devices may affect the observed efficacy of various devices. Although we provided a brief description and training prior to the use of the LifeFlow device, none of the participants had seen or used this device previously, while all had significant clinical experience with rapid fluid administration using the push-pull system. Despite this, the participants were able to achieve improved objective performance with the LifeFlow device. Although meant as a single-use device, to control expenses related to the study, the LifeFlow and the push-pull setup were reused for several trials. The handle on one of the LifeFlow devices broke during use. We do not believe that this was related to overuse as it occurred during the third trial with this particular device and the other devices were used multiple times during the study. However, the device is intended for single-patient use and not multiple uses as in this trial. Alternatively, the break may have been due to user error or excessive force influenced by the competitive nature of the trials. The participants had a self-imposed internal competition to see who could complete the fluid administration goal in the shortest period of time.

Despite the decreased administration time with the LifeFlow device, we did not find subjective improvements in fatigue, physical demand, or pain when comparing the LifeFlow device to the push-pull system. We postulate that any improvement in these subjective parameters may be associated with increased administration time. The aggressive administration of fluid noted in our simulated trial with the potential to deliver a 20 $\mathrm{mL} / \mathrm{kg}$ fluid bolus to a $20 \mathrm{~kg}$ patient in little more than $2 \mathrm{~min}$ utes may have negatively impacted the subjective experience of providers using the devices during the study scenario.

We previously demonstrated that blood pump tubing and a pressure bag around the fluid bag significantly reduced the time to administer a fixed quantity of fluid. ${ }^{16}$ As that study used a 16-gauge cannula, a direct comparison of infusion rates to the present results is not feasible. Additionally, our clinical experience suggests that such devices (blood pump tubing and pressure bags) are rarely used outside of the operating room, while the LifeFlow device was specifically developed for rapid fluid administration during resuscitation. The majority of the preliminary data regarding the device are from the intensive care unit, emergency department, and trauma scenarios. Despite this, there is potential for its use for operating room resuscitation, especially if it can be used to deliver blood products and colloids. Regardless of the method used for rapid fluid administration, these devices may create a clinical scenario where air embolism may be a risk. ${ }^{21,22}$ This risk can be expected to increase whenever fluids are rapidly administered under pressure. Both the setups used in the current study would allow the rapid recognition of air being entrained into the system, as it can be seen in the syringe in the LifeFlow device or in the push-pull setup. 


\section{Conclusion}

We found that the LifeFlow device allowed a significant reduction in fluid administration time when compared to a standard push-pull syringe system in a simulated environment; however, increased physical demand with the LifeFlow device was noted with catheter sizes 18 and 22 gauge, and increased fatigue with the LifeFlow device was noted with all catheter sizes. The LifeFlow Rapid Infuser is a novel system that is easy to set up and use, which allows for the rapid administration of fluid. However, we did not note any improvement in subjective measures of fatigue and pain when using the device for rapid fluid administration. Whenever there is the rapid administration of fluid, there is also a concern regarding infiltration of administration sites with the extravasation of fluid. Ongoing observation of the administration site is needed to limit the potential morbidity related to such problems.

\section{Acknowledgments}

We would like to acknowledge the participation of our anesthesiology faculty, fellows, and residents as well as our CRNA and SRNA colleagues without whom we would not have been able to complete our trial. The manufacturer provided the LifeFlow Rapid Infusion Devices used in the current study free of charge. Otherwise, this research did not receive any specific grant from funding agencies in the public, commercial, or not-for-profit sectors.

\section{Author contributions}

Study design (HCG, HW, DT, TB, JDT); data collection (HCG, $\mathrm{HW}$ ); preparation of first draft (HCG); manuscript revisions and reviews (HCG, HW, DT, TB, JDT). All authors contributed toward data analysis, drafting and critically revising the paper and agree to be accountable for all aspects of the work.

\section{Disclosure}

The authors disclose receiving the LifeFlow Rapid Infusion devices used in this study free of charge from the manufacturer. The authors report no other conflicts of interest in this work.

\section{References}

1. Yager P, Noviski N. Shock. Pediatr Rev. 2010;31(8):311-319.

2. Tobias JD. Shock in children: the first 60 minutes. Pediatr Ann. 1996;25(6):330-338.
3. Mckiernan CA, Lieberman SA. Circulatory shock in children: an overview. Pediatr Rev. 2005;26(12):451-460.

4. Carcillo JA, Davis AL, Zaritsky A. Role of early fluid resuscitation in pediatric septic shock. JAMA. 1991;266(9):1242-1245.

5. Han YY, Carcillo JA, Dragotta MA, Bills DM, Watson RS, Westerman ME, Orr RA. Early reversal of pediatric-neonatal septic shock by community physicians is associated with improved outcome. Pediatrics. 2003;112(4):793-799.

6. Paul R, Neuman MI, Monuteaux MC, Melendez E. Adherence to PALS Sepsis Guidelines and Hospital Length of Stay. Pediatrics. 2012;130(2):e273-e280.

7. Davis AL, Carcillo JA, Aneja RK, et al. American College of Critical Care Medicine Clinical Practice Parameters for Hemodynamic Support of Pediatric and Neonatal Septic Shock. Crit Care Med. 2017;45(6):1061-1093.

8. Nolan JP, Soar J, Zideman DA, et al. European Resuscitation Council Guidelines for Resuscitation 2010 Section 1. Executive summary. Resuscitation. 2010;81(10):1219-1276.

9. Brierley J, Carcillo JA, Choong K, et al. Clinical practice parameters for hemodynamic support of pediatric and neonatal septic shock: 2007 update from the American College of Critical Care Medicine. Crit Care Med. 2009;37(2):666-688.

10. Dellinger RP, Levy MM, Rhodes A, et al. Surviving sepsis campaign: international guidelines for management of severe sepsis and septic shock: 2012. Crit Care Med. 2013;41(2):580-637.

11. Toshniwal G, Ahmed Z, Sengstock D. Simulated fluid resuscitation for toddlers and young children: effect of syringe size and hand fatigue. Paediatr Anaesth. 2015;25(3):288-293.

12. Lopez BL, Davis-Moon L, Sterious W, Bulette M, Liang-Ma X, Christopher TA. The Power Infuser: a new device for rapid fluid infusion. $\mathrm{Am}$ J Emerg Med. 2003;21(2):129-132.

13. Aeder MI, Crowe JP, Rhodes RS, Shuck JM, Wolf WM. Technical limitations in the rapid infusion of intravenous fluids. Ann Emerg Med. 1985;14(4):307-310.

14. Stoner MJ, Goodman DG, Cohen DM, Fernandez SA, Hall MW. Rapid fluid resuscitation in pediatrics: testing the American College of Critical Care Medicine Guideline. Ann Emerg Med. 2007;50(5):601-607.

15. Kamata M, Walia H, Hakim M, Tumin D, Tobias JD. An in vitro assessment of the efficacy of various IV cannulas for the rapid IV fluid administration. Pediatr Crit Care Med. 2017;18(5):e224-e228.

16. Balaban O, Walia H, Tumin D, Bhalla T, Tobias JD. Efficacy of rapid fluid administration using various set-ups and devices. Pediatr Emerg Care. In press 2018

17. Hart SG, Staveland LE. Development of a NASA-TLX (Task Load Index): results of empirical and theoretical research. In: Hancock PS, Meshkati N, editors. Human Mental Workload. Amsterdam: NorthHolland; 1988:139-183.

18. Stoner MJ, Goodman DG, Cohen DM, Fernandez SA, Hall MW. Rapid fluid resuscitation in pediatrics: testing the American College of Critical Care Medicine guideline. Ann Emerg Med. 2007;50(5):601-607.

19. Mcpherson D, Adekanye O, Wilkes AR, Hall JE. Fluid flow through intravenous cannulae in a clinical model. Anesth Analg. 2009;108(4):1198-1202.

20. Reddick AD, Ronald J, Morrison WG. Intravenous fluid resuscitation: was Poiseuille right? Emerg Med J. 2011;28(3):201-202.

21. Orebaugh SL. Venous air embolism: clinical and experimental considerations. Crit Care Med. 1992;20(8):1169-1177.

22. Palmon SC, Moore LE, Lundberg J, Toung T. Venous air embolism: a review. J Clin Anesth. 1997;9(3):251-257. 


\section{Publish your work in this journal}

Medical Devices: Evidence and Research is an international, peerreviewed, open access journal that focuses on the evidence, technology, research, and expert opinion supporting the use and application of medical devices in the diagnosis, monitoring, treatment and management of clinical conditions and physiological processes. The identification of novel devices and optimal use of existing devices which will lead to improved clinical outcomes and more effective patient management and safety is a key feature. The manuscript management system is completely online and includes a quick and fair peer-review system. Visit http://www. dovepress.com/testimonials.php to read real quotes from authors.

Submit your manuscript here: https://www.dovepress.com/medical-devices-evidence-and-research-journal 